\title{
Parameter Space Design of Robust Control Systems
}

\author{
JUERGEN ACKERMANN
}

\begin{abstract}
Find a state or output feedback with fixed gains such that nice stability (defined by a region in the eigenvalue plane) is robust with respect to large plant parameter variations, sensor failures, and quantization effects in the controller. Keep the required magnitude of control inputs small in this design. A tool for tackling such problems by design in the controller parameter space $\mathcal{K}$ is introduced. Pole placement is formulated as an affine map from the space $\mathcal{P}$ of characteristic polynomial coefficients to the $\mathcal{K}$ space. This allows determining the regions in the $\mathcal{K}$ space, which place all eigenvalues in the desired region in the eigenvalue plane. Then tradeoffs among a variety of different design specifications can be made in $\mathcal{K}$ space. The use of this tool is illustrated by the design of a crane control system. Several open research problems result from this approach: graphical computer-aided design of robust systems, algebraic robustness conditions, and algorithms for iterative design of robust control systems.
\end{abstract}

\section{INTRODUCTION}

$\mathrm{N}$ this paper a new tool for the design of robust control 1 systems is proposed. First, the type of robustness problems is described for which the tool can be applied.

Robustness of control systems is defined in terms of a system property which is invariant under a specified class of perturbations. The system property considered in this paper is "nice stability" as specified by a region $\Gamma$ in the eigenvalue plane, in which all eigenvalues have to remain

Manuscript received October 30, 1978; revised July 19, 1979 and March 6, 1980. Paper recommended by D. D. Siljak, Past Chairman of the Large Scale Systems, Differential Games Committee. This work was supported by the Deutsche Forschungs-und Versuchsanstalt fuer Luftund Raumfahrt e. V., by the U.S. Air Force under Grant AFOSR 78-3633, and by the Joint Services Electronics Program under Contract N00014-79-C-0424.

The author is with DFVLR-Institut fuer Dynamik der Flugsysteme, 8031 Oberpfaffenhofen, West Germany. in spite of perturbations. The perturbations may be large changes of physical parameters of the plant, failures of sensors, inaccurate implementation of the control law, or the gain reduction effect of actuator saturation.

The following assumptions are made.

1) Only single input linear plants

$$
\begin{aligned}
\dot{x}(t) & =A \boldsymbol{x}(t)+b u(t) \quad \text { or } \\
\boldsymbol{x}(k+1) & =\boldsymbol{A} \boldsymbol{x}(k)+\boldsymbol{b u}(k) \\
\boldsymbol{x}^{T} & =\left[x_{1} \cdots x_{n}\right]
\end{aligned}
$$

are considered. It is assumed that (1) is written in "sensor coordinates," i.e., if originally an output equation $y=C x$ for $s$ independent measurements, rank $C=s \leqslant n$ was given, the system has been first transformed such that $y$ becomes part of the state vector.

2) $\boldsymbol{A}$ and $\boldsymbol{b}$ may depend on a physical parameter vector $\boldsymbol{\theta}$. Only some typical values

$$
A_{j}=A\left(\theta_{j}\right), \quad b_{j}=b\left(\theta_{j}\right) \quad j=1,2 \cdots J
$$

may be given, e.g., the linearized equations for an aircraft at different altitudes and speeds.

3) The assumed controller structure is state feedback.

$$
u=-\boldsymbol{k}^{T} x, \quad \boldsymbol{k}^{T}=\left[k_{1} k_{2} \cdots k_{n}\right] .
$$

Some elements $k_{i}$ may be given, e.g., they may be zero for output feedback. The remaining elements of $\boldsymbol{k}^{T}$ constitute the free design parameters. They are the coordinates of a parameter space $\overline{\mathscr{K}}$ in which the design is performed. 


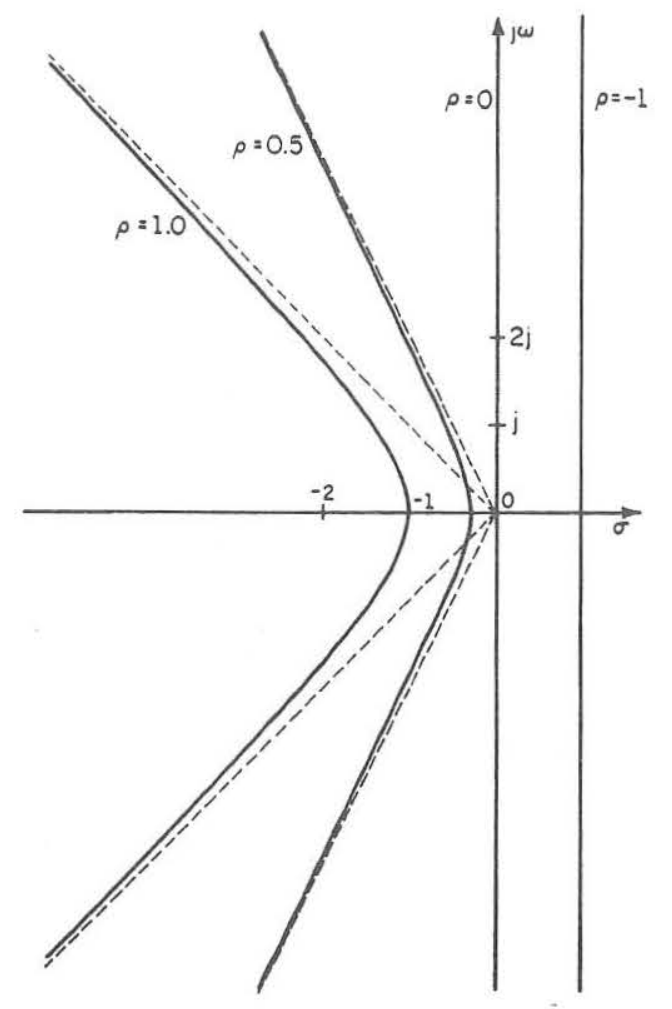

Fig. 1. Hyperbolic boundaries in s-plane.

4) It is assumed that desirable features of the dynamic behavior of the control system can be specified by a region $\Gamma$ in the eigenvalue plane. Examples are

a) the stability region, i.e., the left half $s$ plane or the interior of the unit circle in the $z$ plane;

b) military specifications for damping and minimum and maximum values for the natural frequency of modes of an aircraft [6];

c) the region to the left of the hyperbola $\omega^{2}=\sigma^{2}-1$, $\sigma<-1$ in the $s$-plane, $s=\sigma+j \omega$. This guarantees $1 / \sqrt{2}$ damping and a real part smaller than -1 for all eigenvalues. Also other hyperbolas may be used; see Fig. 1. This region may be bounded to the left by a large circle around $s=0$ in order to achieve a bandwidth limitation.

d) Another example is the interior of one of the circles of Fig. 2 in the $z$ plane.

A control system with all eigenvalues in $\Gamma$ will be called "nicely stable." The problem is to find the free parameters in $\boldsymbol{k}^{T}$ such that all roots of

$$
\prod_{j=1}^{J} \operatorname{det}\left(\lambda \boldsymbol{I}-\boldsymbol{A}_{j}+\boldsymbol{b}_{j} \boldsymbol{k}^{T}\right)=0
$$

are located in $\Gamma$. Three cases can occur.

1) There exists a set of admissible solutions in the space $\overline{\mathcal{K}}$ of free parameters.

2) This set degenerates to one or more single points.

3) No solution exists.

Note that case 2) does occur only if the region is closed, i.e., the boundary itself belongs to the region. This makes a theoretical difference if the stability boundary is mapped into $\overline{\mathscr{K}}$ space. Practically, however, smaller regions $\Gamma$ inside the stability region will be chosen, and cases where one or more eigenvalues are located on the boundary and all others inside $\Gamma$ will be considered admissible.

One possibility to formulate a well-posed design problem is to imbed $\Gamma$ into a family $\Gamma_{r}$ of boundaries and to find the best value of the parameter $r$ for which case 2) occurs. This procedure does not allow taking other design specifications into consideration. Therefore, here $\Gamma$ is fixed and it is assumed that in case 3) a richer controller structure must be assumed, e.g., dynamic linear feedback or gain scheduling, or the specified region $\Gamma$ must be relaxed. In case 1) a design point can be chosen from the admissible set on the basis of additional requirements, e.g.,

a) constraints on $|u|$ or $|\dot{u}|$;

b) robustness of $\Gamma$ with respect to nonideal control law implementation;

c) robustness of a relaxed "emergency specification" $\Gamma_{E}$ with respect to sensor failures;

d) points from the admissible set may be used in the simulation of the control system with a more complex plant model including nonlinearities, structural modes, coupling terms, etc., which had been neglected in the first design step.

The development of the design concept in this paper is structured as follows.

1) The eigenvalue constraint $\Gamma$ is first represented as a region in the parameter space $\mathscr{P}$ of coefficients of the characteristic polynomial

$$
\begin{aligned}
P(\lambda) & =p_{0}+p_{1} \lambda+\cdots+p_{n-1} \lambda^{n-1}+\lambda^{n} \\
& =\left[\begin{array}{ll}
\boldsymbol{p}^{T} & 1
\end{array}\right]\left[\begin{array}{c}
1 \\
\lambda \\
\vdots \\
\lambda^{n}
\end{array}\right]=\prod_{i=1}^{n}\left(\lambda-\lambda_{i}\right) .
\end{aligned}
$$

In other words, a region $P_{\Gamma}$ in $\mathscr{P}$ is determined such that

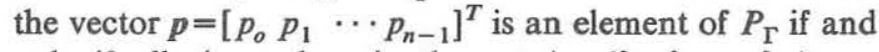
only if all eigenvalues in the set $\Lambda=\left\{\lambda_{1}, \lambda_{2} \cdots \lambda_{n}\right\}$ are located in $\Gamma$.

2) By pole assignment a given controllable pair $A, b$ defines an affine mapping $\boldsymbol{k}^{T}=\boldsymbol{k}_{0}^{T}+\boldsymbol{p}^{T} W^{-1}$ from $\mathcal{P}$ to $\mathscr{K}$ where $\mathcal{K}$ is the parameter space of full state feedback (3), i.e., the coordinates of $\mathcal{K}$ are $k_{1}, k_{2} \cdots k_{n}$. The region $P_{\Gamma}$ is mapped into a region $K_{\Gamma}$ and $p \in P_{\Gamma} \Leftrightarrow k \in K_{\Gamma}$.

3) For $J$ pairs $\boldsymbol{A}_{j}, \boldsymbol{b}_{j}$ the intersection of $K_{\Gamma 1}, K_{\Gamma 2} \cdots K_{\Gamma J}$ is the set of solutions $K_{\Gamma}$ to (4).

4) Fixing some gains $k_{i}$ defines a subspace $\overline{\mathcal{K}}$ of $\mathscr{K}$. Its intersection with $K_{\Gamma}$ is the admissible set $\bar{K}_{\Gamma}$ of solutions under this constraint.

5) Additional design constraints are interpreted in terms of $\boldsymbol{k}$ and $\bar{K}_{\Gamma}$.

6) This concept is the basis for both graphic and algebraic computer-aided design methods and algorithms for robust control systems. 
Parameter space methods have a long tradition, mainly in Russia and Yugoslavia. Šiljak [1, ch. 1, 2] gives a historical review of the work by Vishnegradsky, Neimark, Mitrovic, and himself. In these methods an arbitrary controller structure with free parameter vector $\boldsymbol{k}$ can be assumed. The characteristic polynomial

$$
P(\lambda)=\sum_{i=0}^{n} p_{i}(k) \lambda^{i}=0
$$

defines a map $\mathscr{K} \rightarrow \mathcal{P}$ which has to be inverted in order to map boundaries from $\mathcal{P}$ to $\mathcal{K}$. In this paper the controller structure is restricted to state feedback (3), and the map $\mathcal{P} \rightarrow \mathcal{K}$ is determined directly via pole assignment. The following diagram summarizes the relationships between $\Lambda, \mathcal{P}$, and $\mathcal{K}$ for state feedback.

$$
\Lambda \underset{\begin{array}{c}
\text { numerical } \\
\text { factorization }
\end{array}}{\stackrel{P(\lambda)=\pi\left(\lambda-\lambda_{i}\right)}{\rightleftarrows}} \mathcal{P} \underset{P(\lambda)=\operatorname{det}\left(\lambda I-A+b k^{T}\right)}{\stackrel{k^{T}=k_{0}^{T}+p^{T} W^{-1}}{\rightleftarrows}} \mathcal{K}
$$

Apparently it is easier to go in the direction $\Lambda \rightarrow \mathscr{P} \rightarrow \mathscr{K}$ than in the opposite direction. In other words, it is easier to study the effect of one eigenvalue $\lambda_{i}$ on $\boldsymbol{k}^{T}$ than to study the effect of one gain $k_{i}$ on $\Lambda$.

\section{Representing an Eigenvalue Region $\Gamma$ as a Characteristic Polynomial Coefficient Region $P_{\Gamma}$}

Let the region $\Gamma$ in the $\lambda$-plane $\lambda=v+j w$ be bounded and symmetric with respect to the real axis. The boundary

$$
w^{2}=w^{2}(v)
$$

has intersections $v_{R i}, i=1,2 \cdots 2 N$, with the real axis $w^{2}\left(v_{R i}\right)=0$.

Assume that the eigenvalues are varied continuously until one or more of them cross the boundary (7). This may occur for a real pole at $v_{R}, w^{2}\left(v_{R}\right)=0$, or for a complex pair of poles $v \pm j w$ on the boundary (7).

\section{A. Real Root Boundary}

For a real root at $\lambda=v_{R}$

$$
\begin{aligned}
& P(\lambda)=\left(\lambda-v_{R}\right) \cdot R(\lambda), \\
& R(\lambda)=r_{0}{ }^{+} r_{1} \lambda+\cdots+r_{n-2} \lambda^{n-2}+\lambda^{n-1} .
\end{aligned}
$$

The coefficients $p_{i}$ are linear in the remaining $n-1$ free parameters $r_{0}, r_{1} \cdots r_{n-2}$, i.e., the boundary in $\odot$ space is an $(n-1)$-dimensional hyperplane. Its equation is

$$
P\left(v_{R}\right)=0 .
$$

\section{B. Complex Root Boundary}

For a complex pair $\lambda=v \pm j w$ on the boundary (7)

$$
P(\lambda)=Q(\lambda) R(\lambda)
$$

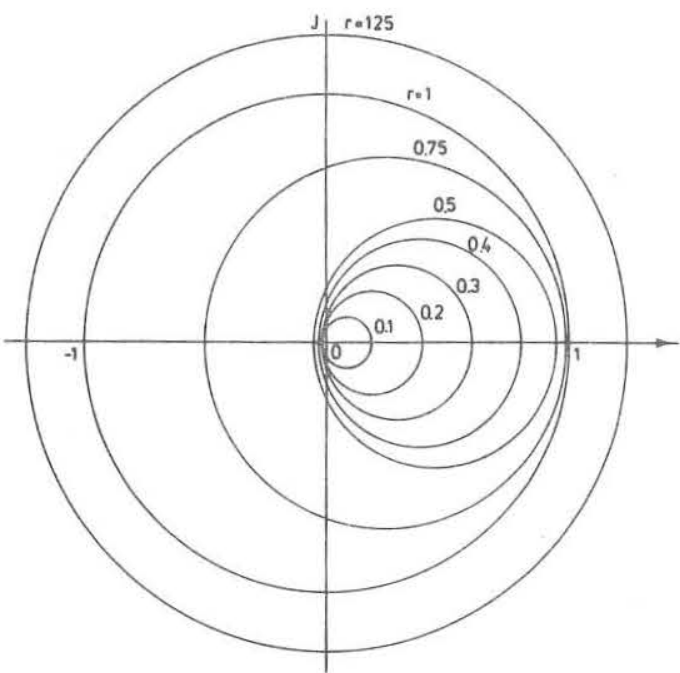

Fig. 2. Circular boundaries in $z$-plane.

$$
\begin{aligned}
Q(\lambda) & =(\lambda-v-j w)(\lambda-v+j w) \\
& =\lambda^{2}-2 v \lambda+v^{2}+w^{2}(v) \\
R(\lambda) & =r_{0}+r_{1} \lambda+\cdots+r_{n-3} \lambda^{n-3}+\lambda^{n-2} .
\end{aligned}
$$

The coefficients $p_{i}$ depend nonlinearly on the $n-1$ free parameters $v, r_{o} \cdots r_{n-3}$. However, for a fixed $v$, i.e., one fixed complex pair on the boundary, they are linear and the boundary is an $(n-2)$-dimensional hyperplane. As $v$ varies, this hyperplane moves and forms the complex root boundary. Another way of viewing this same surface is to keep the $n-2$ eigenvalues in $R(\lambda)$ fixed and to move the complex pair along the boundary. This generates a onedimensional curve $\boldsymbol{p}(v)$. The shape of this curve depends on the boundary $w^{2}(v)$.

Some boundaries of particular interest are as follows.

1) Imaginary axis, stability boundary for $\lambda=s$.

$$
\begin{gathered}
v=0, \quad Q(\lambda)=\lambda^{2}+w^{2}, \\
p \text { linear in } w^{2} \text { for fixed } R(\lambda) .
\end{gathered}
$$

2) Parallel to imaginary axis

$$
\begin{gathered}
v=v_{1}, \quad Q(\lambda)=\lambda^{2}-2 v_{1} \lambda+v_{1}^{2}+w^{2}, \\
p \text { linear in } w^{2} \text { for fixed } R(\lambda) .
\end{gathered}
$$

3) Conic section, symmetric to the real axis, i.e.,

$$
w^{2}=c_{0}+c_{1} v+c_{2} v^{2} .
$$

Some special cases follow.

$c_{2}<0$, ellipse: of particular interest are circles $c_{2}=-1$, constant natural frequency curves in the $s$-plane, stability limit, and other boundaries in the $z$-plane. See Fig. 2.

$c_{2}=0$, parabola, or if also $c_{1}=0, c_{o}>0$, straight line parallel to real axis. For $c_{o}=c_{1}=c_{2}=0$ real axis, i.e., boundary between real and complex eigenvalues.

$c_{2}>0$, hyperbola: in particular two straight lines for $w^{2}=c_{2}\left(v-v_{0}\right)^{2}, c_{2}>0$, e.g., constant damping lines in the $s$-plane. This boundary is frequently combined with a 


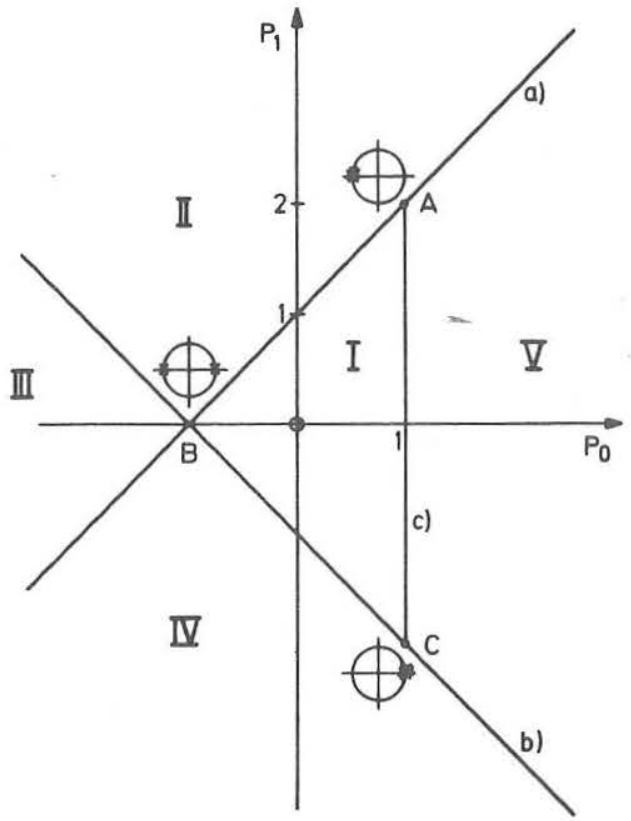

Fig. 3. Real and complex root boundaries partition the $\Phi$ plane into five regions. $A B C$ is the stability triangle for second-order discrete-time systems.

parallel to the imaginary axis. Here it is more convenient to use a hyperbola, which guarantees the required damping and minimum negative real part of the eigenvalues. See Fig. 1.

Substituting (11) into $Q(\lambda)$

$$
Q(\lambda)=\lambda^{2}-2 v \lambda+\left(1+c_{2}\right) v^{2}+c_{1} v+c_{0} .
$$

Variable $p$ is quadratic in $v$ for fixed $R(\lambda)$. It becomes linear if and only if $c_{2}=-1$, i.e., for a circular boundary in $\lambda$ plane. In other words, if the $n-2$ roots of $R(\lambda)$ are fixed and the remaining two roots of $P(\lambda)$ move as a conjugate pair along any circle in the $\lambda$-plane with center $\lambda_{o}$ on the real axis and radius $r$, then the corresponding point in $\mathscr{P}$ space moves along a straight line. Its endpoints correspond to double eigenvalues at the axis intersections $\lambda_{L}=\lambda_{o}-r$ and $\lambda_{R}=\lambda_{o}+r$ of the circle, i.e., $Q_{L}(\lambda)=(\lambda-$ $\left.\lambda_{L}\right)^{2}$ and $Q_{R}(\lambda)=\left(\lambda-\lambda_{R}\right)^{2}$. The polynomials $P_{L}(\lambda)=$ $Q_{L}(\lambda) R(\lambda)$ and $P_{R}(\lambda)=Q_{R}(\lambda) R(\lambda)$ represent the endpoints of this straight line segment in the $\mathcal{P}$ space.

For second- and third-order systems it is possible to visualize regions in $\mathscr{P}$ space graphically. This is done in the following for the unit circle in the $\lambda=z$ plane, i.e., the stability region of discrete systems in the $\mathcal{P}$ space is determined.

For $n=2$

a) Real root boundary for $z=1$. By (9) the boundary is the straight line $P(1)=p_{o}+p_{1}+1=0$.

b) Real root boundary for $z=-1, P(-1)=p_{o}-p_{1}+1$ $=0$.

c) Complex root boundary $v^{2}+w^{2}=1,-1<v<1$. In (10) $R(\lambda)=1, \quad P(\lambda)=Q(\lambda)=\lambda^{2}-2 v \lambda+1, \quad p_{o}=1, p_{1}=$ $-2 v,-2<p_{1}<2$.

Fig. 3 shows the three boundaries. At $B$ the two real root boundaries intersect, i.e., $P_{B}(z)=(z-1)(z+1), p_{B}^{T}=$

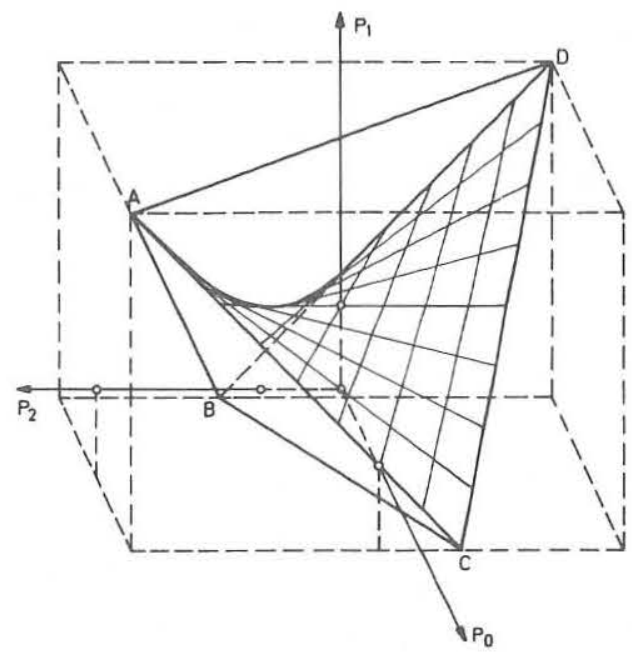

Fig. 4. Stability region for third-order discrete-time systems.

[-1 0]. Similarly $P_{A}(z)=(z+1)^{2}$, i.e., $\boldsymbol{p}_{A}^{T}=\left[\begin{array}{ll}1 & 2\end{array}\right]$ and $P_{C}(z)=(z-1)^{2}$, i.e., $p_{C}^{T}=\left[\begin{array}{ll}1 & -2\end{array}\right]$. The boundaries a), b), and c) partition the $p_{o}-p_{1}$-plane into five regions such that I) both poles are inside the unit circle; II) one left, one inside; III) one left, one right; IV) one inside, one right; V) complex outside or both left or both right [a distinction between these three cases in region V) would require a further boundary distinguishing real and complex roots]. Usually only the stability region I is of interest.

For $n=3$, only the stability region is shown in Fig. 4. Its real root boundaries are the triangles $A B C$ in the plane $P(-1)=0$ and $B C D$ in the plane $P(1)=0$. The complex boundary is obtained by $(10), P(\lambda)=\left(\lambda^{2}-2 v \lambda+1\right)\left(r_{0}+\right.$ $\lambda)$. For a fixed value of $r_{o}$ it is a straight line from a point on $B A(v=-1)$ to a point on $D C(v=1)$. For a fixed value of $v$ it is a straight line from a point on $A C\left(r_{o}=1\right)$ to a point on $B D\left(r_{o}=-1\right)$. This second-order surface is a hyperbolic paraboloid. It has a saddle point at $\boldsymbol{p}^{T}=\left[\begin{array}{ll}0 & 1\end{array}\right.$ 0]. The stability region is uniquely determined by its vertices $A, B, C$, and $D$ which correspond to the four polynomials with zeros in the set $\{-1,1\}$, i.e., $\boldsymbol{p}_{A}^{T}=\left[\begin{array}{ll}1 & 3\end{array}\right.$ 3], $\boldsymbol{p}_{B}^{T}=\left[\begin{array}{lll}-1 & -1 & 1\end{array}\right], \boldsymbol{p}_{C}^{T}=\left[\begin{array}{lll}1 & -1 & -1\end{array}\right]$, and $\boldsymbol{p}_{D}^{T}=\left[\begin{array}{ll}-1 & \end{array}\right.$ 3 - 3]. It is seen from Fig. 4 that the tetrahedron $A B C D$ is the convex hull of the stability region.

Fam and Meditch [2] showed that this property generalizes to arbitrary degree $n$ of the characteristic polynomial. For an $n$ th-order linear discrete system the convex hull of the stability region in $\mathcal{P}$ space is a polyhedron whose vertices correspond to the $n+1$ polynomials with zeros in the set $\{-1,1\}$.

Let the vertices be ordered such that the vertex vector $\boldsymbol{p}_{i}$ corresponds to the polynomial $P_{i}=(z-1)^{n-i}(z+1)^{i}, i=$ $0,1,2 \cdots n$. The $n+1$ vertices are independent, i.e., the vectors $\boldsymbol{p}_{1}-\boldsymbol{p}_{o}, \boldsymbol{p}_{2}-\boldsymbol{p}_{o} \cdots \boldsymbol{p}_{n}-\boldsymbol{p}_{o}$ are linearly independent. Therefore, any vector $\boldsymbol{p}$ can be uniquely expressed as

$$
p=\sum_{i=0}^{n} \mu_{i} p_{i}
$$


with

$$
\sum_{i=0}^{n} \mu_{i}=1
$$

$\mu_{i}$ are called "barycentric coordinates" of $\boldsymbol{p}$ [3]. Variable $\boldsymbol{p}$ may be visualized as the center of mass of the polyhedron if a unit mass may be distributed arbitrarily over the $n+1$ vertices. As long as the masses $\mu_{i}$ are positive the center of mass is inside the polyhedron. The interior of the polyhedron is an $n$-dimensional simplex. It is characterized by the fact that the barycentric coordinates are nonnegative $\mu_{i} \geqslant 0, i=0,1 \cdots n$. Equations (13) and (14) may be written

$$
\left[\begin{array}{cccc}
\boldsymbol{p}_{0} & \boldsymbol{p}_{1} & \cdots & \boldsymbol{p}_{n} \\
1 & 1 & \cdots & 1
\end{array}\right]\left[\begin{array}{c}
\mu_{0} \\
\mu_{1} \\
\vdots \\
\mu_{n}
\end{array}\right]=\left[\begin{array}{c}
\boldsymbol{p} \\
1
\end{array}\right]
$$

where $p$ belongs to the simplex if and only if all elements of

$$
\mu=\left[\begin{array}{cccc}
p_{0} & p_{1} & \cdots & p_{n} \\
1 & 1 & \cdots & 1
\end{array}\right]^{-1}\left[\begin{array}{l}
p \\
1
\end{array}\right]
$$

are nonnegative (or positive if we consider the open region, which corresponds to the interior of the unit circle in the $z$-plane without the circle itself). Equation (16) gives the strongest linear necessary conditions for stability of a discrete system.

It follows from (12) with $c_{2}=-1$ that the convex hull property generalizes to arbitrary circles with radius $r$ and center $v_{o}$ on the real axis of the eigenvalue plane. This circle intersects the real axis at $v_{L}=v_{o}-r$ and $v_{R}=v_{o}+r$. The vertices of the convex hull of the corresponding region in $\mathscr{P}$ space are determined by the $n+1$ polynomials with zeros in the set $\left\{v_{L}, v_{R}\right\}$. This may also be shown by reducing this problem to the previous one via $z^{\prime}=(z-$ $\left.v_{o}\right) / r$. It is, therefore, convenient to specify the eigenvalue region by one member of the family of nonintersecting circles $\Gamma_{r}$ shown in Fig. 2. Its equation is

$$
\begin{array}{rlrl}
\left(v-v_{0}\right)^{2}+w^{2} & =r^{2} & & \\
v_{0}\left(v_{0}-1\right) & =0.99 r(r-1), & v_{0}<0.5 \text { for } r<1 \\
v_{0} & =0 & \text { for } r \geqslant 1 .
\end{array}
$$

For $r=0$ it is the deadbeat solution with all eigenvalues at $z=0$. With increasing $r$, the center $v_{0}$ of the circles moves to the right until it reaches 0.45 for $r=0.5$ where it then goes back to zero to produce the unit circle for $r=1$. If boundaries in the unstable region are needed, concentric circles with radius $r$ may be used. Circles with radius $r$ in the range $0.3-0.5$ approximate the usual logarithmic spirals for constant damping augmented by a constraint on $|z|$. This eigenvalue region corresponds to well-damped transients. The right shift of the circles excludes heavily oscillatory solutions. Faster solutions with $r \rightarrow 0$ typically require larger control inputs $|u|$ and allow less robustness with respect to plant parameter variations.

The family of circles (17) builds a bridge between the practical aspect, that most newly designed control systems are digital, and the theoretical aspect, that (12) is linear in $v$ for circles only.

For continuous-time systems the family of hyperbolas $\Gamma_{\rho}$ of Fig. 1 in the $s(=\sigma+j \omega)$-plane may be used. Its equation is

$$
\begin{aligned}
\omega^{2} & =-\rho^{2}+\sigma^{2} / \rho^{2} \quad \text { for } \sigma<0 \\
\sigma & =-\rho \quad \text { for } \sigma>0 .
\end{aligned}
$$

For large $\rho$ an extremely fast solution is obtained: $\rho=1$ gives the $1 / \sqrt{2}$ damping line as asymptotes; for $\rho \rightarrow 0$ it goes to the imaginary axis. Negative $\rho$ represent parallels to the imaginary axis in the right half plane.

\section{MAPPIng From $\mathcal{P}$ SPACE to $\mathscr{K}$ SPAce (Pole Assignment)}

A controllable pair $(\boldsymbol{A}, \boldsymbol{b})$ defines via pole assignment a mapping from $\mathcal{P}$ to $\mathscr{K}$ space. This mapping is affine, i.e., it consists of a translation and a nonsingular linear mapping

$$
\boldsymbol{k}^{T}=\boldsymbol{k}_{0}^{T}+\boldsymbol{p}^{T} \boldsymbol{W}^{-1} .
$$

The foundation of this mapping is Theorem 1.

Theorem 1 (Pole Assignment): Given a polynomial $P(\lambda)$ $=p_{o}+p_{1} \lambda+\cdots+p_{n-1} \lambda^{n-1}+\lambda^{n}=\left[\begin{array}{ll}\boldsymbol{p}^{T} & 1\end{array}\right] \lambda, \lambda=\left[\begin{array}{lll}1 & \lambda & \cdots\end{array}\right.$ $\left.\lambda^{n}\right]^{T}$, an $n \times n$ matrix $\boldsymbol{A}$, and an $n \times 1$ vector $\boldsymbol{b}$ such that $\operatorname{det} \boldsymbol{R} \neq 0, \boldsymbol{R}=\left[\boldsymbol{b}, \boldsymbol{A b} \cdots \boldsymbol{A}^{n-1} \boldsymbol{b}\right]$. The unique solution to

$$
\operatorname{det}\left(\lambda \boldsymbol{I}-\boldsymbol{A}+\boldsymbol{b} \boldsymbol{k}^{T}\right)=\left[\begin{array}{ll}
\boldsymbol{p}^{T} & 1
\end{array}\right] \boldsymbol{\lambda}
$$

is

$$
\boldsymbol{k}^{T}=\left[\begin{array}{ll}
\boldsymbol{p}^{T} & 1
\end{array}\right] \boldsymbol{E}, \quad \boldsymbol{E}=\left[\begin{array}{c}
\boldsymbol{e}^{T} \\
\boldsymbol{e}^{T} \boldsymbol{A} \\
\vdots \\
\boldsymbol{e}^{T} \boldsymbol{A}^{n}
\end{array}\right]
$$

where $e^{T}$ is the last row of $R^{-1}$.

Proof: Existence and uniqueness of the solution were shown by Rissanen [4] by transformation to control canonical form. The solution in form of (20) is derived as follows.

Let $\boldsymbol{F}=\boldsymbol{A}-\boldsymbol{b} \boldsymbol{k}^{\prime}$ and expand powers of $\boldsymbol{F}$ in terms of the form $\boldsymbol{A}^{i} \boldsymbol{b} \boldsymbol{k}^{T} \boldsymbol{F}^{j}$.

$$
\begin{aligned}
F^{0}= & A^{0}=I \\
F= & A-b k^{T} \\
F^{2}= & A^{2}-A b k^{T}-b k^{T} F \\
& \vdots \\
F^{n}= & A^{n}-A^{n-1} b k^{T}-A^{n-2} b k^{T} F \\
& -\cdots-b k^{T} F^{n-1}
\end{aligned}
$$

Multiply the first equation by $p_{o}$, the second by $p_{1}$, etc., 
the $(n+1)$ st row by one, and add the equations

$$
P(\boldsymbol{F})=P(A)-\left[\boldsymbol{b}, \boldsymbol{A b} \cdots A^{n-1} \boldsymbol{b}\right]\left[\begin{array}{c}
\vdots \\
\boldsymbol{k}^{T}
\end{array}\right] .
$$

By Cayley-Hamilton $P(F)=0$. Then

$$
\left[\begin{array}{c}
\vdots \\
\boldsymbol{k}^{T}
\end{array}\right]=\boldsymbol{R}^{-1} P(A), \quad \boldsymbol{R}=\left[\boldsymbol{b}, A b \cdots A^{n-1} \boldsymbol{b}\right]
$$

$\boldsymbol{k}^{T}$ must satisfy the last row, i.e.,

$$
k^{T}=e^{T} P(A), \quad e^{T}=\left[\begin{array}{llll}
0 & \cdots & 0 & 1
\end{array}\right] \boldsymbol{R}^{-1} .
$$

Explicitly

$$
\begin{aligned}
& \boldsymbol{k}^{T}=e^{T}\left[p_{0} I+p_{1} \boldsymbol{A}+\cdots+p_{n-1} A^{n-1}+\boldsymbol{A}^{n}\right] \\
& \boldsymbol{k}^{T}=\left[\begin{array}{lllll}
p_{0} & p_{1} & \cdots & p_{n-1} & 1
\end{array}\right]\left[\begin{array}{c}
e^{T} \\
e^{T} A \\
\vdots \\
e^{T} A^{n}
\end{array}\right] \quad \text { Q.E.D. }
\end{aligned}
$$

The form (22) of the result was derived in [5]. Equation (23) may be rewritten in the form of (18) with

$$
k_{0}^{T}=e^{T} A^{n}, \quad W^{-1}=\left[\begin{array}{l}
e^{T} \\
e^{T} A \\
\vdots \\
e^{T} A^{n-1}
\end{array}\right]
$$

Note that $W$ is the matrix that transforms $A, b$ to the control canonical form

$$
\begin{aligned}
\boldsymbol{W}^{-1} A \boldsymbol{W} & =\left[\begin{array}{cccc}
0 & 1 & & 0 \\
& & \ddots & \\
-a_{0} & \cdots & & -a_{n-1}
\end{array}\right], \\
\boldsymbol{W}^{-1} \boldsymbol{b} & =\left[\begin{array}{c}
0 \\
\vdots \\
0 \\
1
\end{array}\right] .
\end{aligned}
$$

This is a more popular way of doing pole assignment. It was shown in [5] that the columns $w_{i}$ of $W=\left[\begin{array}{lll}w_{1} & \cdots & w_{n}\end{array}\right]$ can be determined recursively by Leverrier's algorithm:
This describes the mapping from $\mathcal{K}$ to $\mathscr{P}$ as

$$
\begin{aligned}
& \boldsymbol{p}^{T}=\left(\boldsymbol{k}^{T}-\boldsymbol{k}_{0}^{T}\right) W=\boldsymbol{k}^{T} W+\boldsymbol{a}^{T}, \\
& a^{T}=\left[\begin{array}{llll}
a_{0} & a_{1} & \cdots & a_{n-1}
\end{array}\right] .
\end{aligned}
$$

Equations (26) and (27) also provide an alternative way to determine the pole placement matrix $\boldsymbol{E}$ in (20) as

$$
E=\left[\begin{array}{c}
W^{-1} \\
-a^{T} W^{-1}
\end{array}\right]
$$

In numerical calculations of $\boldsymbol{E}$ with large $n$ the accuracy of the vectors $e^{T} A^{i}, i=1,2, \cdots, n$ must be checked. One test is to let $p_{0}=p_{1} \cdots=p_{n-1}=0$. Then $\boldsymbol{k}^{T}=e^{T} A^{n}$. Evaluate $\operatorname{det}\left(\lambda \boldsymbol{I}-\boldsymbol{A}+\boldsymbol{b} \boldsymbol{e}^{T} \boldsymbol{A}^{n}\right)=\tilde{p}_{0}+\tilde{p}_{1} \lambda+\cdots+\tilde{p}_{n-1} \lambda^{n-1}+\lambda^{n}$. The $\tilde{p}_{j}$ should ideally be zero. Their magnitude is a measure for the error in $\boldsymbol{e}^{T} \boldsymbol{A}^{n}$. Another convenient test follows from the definition of $e^{T}$ :

$$
\boldsymbol{e}^{T} \boldsymbol{A}^{i} \boldsymbol{b}= \begin{cases}0 & i=0,1, \cdots, n-2 \\ 1 & i=n-1 .\end{cases}
$$

It is easily seen that this property is also preserved for the closed loop

$$
e^{T}\left(A-b k^{T}\right)^{i} b= \begin{cases}0 & i=0,1, \cdots n-2 \\ 1 & i=n-1\end{cases}
$$

This relation also implies that the vectors $e^{T}, e^{T} A \cdots$ $e^{T} A^{n-1}$ are invariant under state feedback $(A, b) \rightarrow(A-$ $\left.\boldsymbol{b} \boldsymbol{k}^{T}, \boldsymbol{b}\right)$. They are a complete set of invariants of this feedback equivalence class since they uniquely determine a realization in control canonical form with undetermined characteristic polynomial.

The $(n+1) \times n$ pole placement matrix $\boldsymbol{E}$ is a convenient representation of a controllable pair $\boldsymbol{A}, \boldsymbol{b}$, i.e., the element $\boldsymbol{k}=0$ of the feedback equivalence class. The general element may be characterized as

$$
E_{k}=\left[\begin{array}{l}
e^{T} \\
e^{T} A \\
\vdots \\
e^{T} A^{n-1} \\
e^{T} A^{n}-k^{T}
\end{array}\right] .
$$

The mapping of a point $\boldsymbol{p}$ in $\mathscr{P}$ to a point $\boldsymbol{k}$ in $\mathscr{K}$ via $\boldsymbol{k}^{T}=\left[\begin{array}{ll}\boldsymbol{p}^{T} & 1\end{array} \boldsymbol{E}\right.$ requires only $n^{2}$ multiplications and $n^{2}$ additions. This compares favorably with mapping a trial

$$
\begin{aligned}
& \boldsymbol{B}_{0}=\boldsymbol{I} \quad \boldsymbol{w}_{n}=\boldsymbol{B}_{0} \boldsymbol{b} \\
& a_{n-1}=-\operatorname{tr} A \boldsymbol{B}_{0} \quad \boldsymbol{B}_{1}=A \boldsymbol{B}_{0}+a_{n-1} \boldsymbol{I} \quad \boldsymbol{w}_{n-1}=\boldsymbol{B}_{1} \boldsymbol{b} \\
& a_{n-2}=-\frac{1}{2} \operatorname{tr} \boldsymbol{A B}_{1} \quad \boldsymbol{B}_{2}=\boldsymbol{A} \boldsymbol{B}_{1}+a_{n-2} \boldsymbol{I} \quad w_{n-2}=\boldsymbol{B}_{2} \boldsymbol{b} \\
& \text { ¿ } \quad \vdots \quad \vdots \\
& a_{1}=\frac{-1}{n-1} \operatorname{tr} \boldsymbol{A B}_{n-2} \quad \boldsymbol{B}_{n-1}=\boldsymbol{A} \boldsymbol{B}_{n-2}+a_{1} \boldsymbol{I} \quad \boldsymbol{w}_{1}=\boldsymbol{B}_{n-1} \boldsymbol{b} \\
& a_{0}=\frac{-1}{n} \operatorname{tr} A B_{n-1} \quad O=A B_{n-1}+a_{0} I \text { (check). }
\end{aligned}
$$


design point from the parameter space of quadratic criteria via the Riccati equation into $\mathscr{K}$ space. This is an advantage for computer-aided design methods in which many trial design points have to be mapped and displayed graphically.

Example 1 (Pole Assignment): Consider the crane of Fig. 5 with the physical parameters $m_{c}=$ crab mass, $m_{L}=$ load mass, $l=$ rope length, and $g=$ gravitational constant. Its state variables are $x_{1}=$ crab position, $x_{2}=$ crab velocity, $x_{3}=$ rope angle, and $x_{4}=$ rope angular velocity. Input $u$ is the force accelerating the crab. For small rope angles the linearized state equations are

$$
\dot{\boldsymbol{x}}=\left[\begin{array}{cccc}
0 & 1 & 0 & 0 \\
0 & 0 & m_{L} g / m_{c} & 0 \\
0 & 0 & 0 & 1 \\
0 & 0 & -\omega_{p}^{2} & 0
\end{array}\right] \boldsymbol{x}+\frac{1}{m_{c}}\left[\begin{array}{c}
0 \\
1 \\
0 \\
-1 / l
\end{array}\right] u
$$

with $\omega_{p}^{2}=\left(m_{c}+m_{L}\right) g / m_{c} l$. Eigenvalues are $\{0,0$, $\left.j \omega_{p},-j \omega_{p}\right\}$. Then by $(20)$

$$
\begin{aligned}
& \boldsymbol{E}= {\left[\begin{array}{cccc}
l m_{c} / g & 0 & l^{2} m_{c} / g & 0 \\
0 & l m_{c} / g & 0 & l^{2} m_{c} / g \\
0 & 0 & -l m_{c} & 0 \\
0 & 0 & 0 & -l m_{c} \\
0 & 0 & \left(m_{c}+m_{L}\right) g & 0
\end{array}\right] } \\
& k_{1}=p_{0} l m_{c} / g \\
& k_{2}=p_{1} l m_{c} / g \\
& k_{3}=p_{0} l^{2} m_{c} / g-p_{2} l m_{c}+\left(m_{c}+m_{L}\right) g \\
& k_{4}=l m_{c}\left(p_{1} l / g-p_{3}\right) .
\end{aligned}
$$

This result admits some conclusions about static output feedback and gain scheduling

1) for stability $p_{o}>0$, i.e., $k_{1}>0$;

2) for stability $p_{1}>0$, i.e., $k_{2}>0$;

3) $k_{3}=0$ implies the constraint

$$
p_{2}=p_{0} l / g+\left(m_{c}+m_{L}\right) g / l m_{c} ;
$$

4) $k_{4}=0$ implies the constraint

$$
p_{3}=p_{1} l / g
$$

5) a gain scheduling for different known loads $m_{L}$ can be implemented as

$$
k_{3}=k_{30}+m_{L} g \text { with } k_{30}=p_{o} l^{2} m_{c} / g-p_{2} l m_{c}+m_{c} g .
$$

the cher $k_{i}$ remain unchanged. With this gain scheduling a-loop eigenvalues do not depend on the load.

\section{Design In $\mathscr{K}$ SPAce}

\footnotetext{
A. Pole Region Assignment

The mapping of (18) or (20) from $\mathscr{P}$ space to $\mathcal{K}$ space is
used now to $\mathrm{m}_{\text {ap desired regions } P_{\Gamma} \text { into the correspond- }}$
}

ing regions $K_{\Gamma}$. Since the mapping is affine, all straight lines and hyperplanes in the $\mathscr{K}$ space map into straight lines and hyperplanes in $\mathcal{P}$ space. For example, the stability region for third-order discrete systems as shown in Fig. 4 is easily mapped by four pole assignments of the vertices $A, B, C$, and $D$.

Other complex root boundary curves in $\mathcal{P}$ space for noncircular $\Gamma$ may be mapped point by point by (20). The real root boundaries are always $n$-1-dimensional hyperplanes in the $\mathcal{K}$ space.

Example 2 (Design of a Robust Control System):

$$
\begin{aligned}
\boldsymbol{x}(k+1) & =\boldsymbol{A} \boldsymbol{x}(k)+\boldsymbol{b} u(k), \\
A & =\left[\begin{array}{rr}
0 & -4 \\
1 & 4
\end{array}\right] \quad b=\frac{1}{16}\left[\begin{array}{r}
6 \\
-5
\end{array}\right] \\
\boldsymbol{k}^{T} & =\left[\begin{array}{lll}
p_{0} & p_{1} & 1
\end{array}\right]\left[\begin{array}{rr}
5 & 6 \\
6 & 4 \\
4 & -8
\end{array}\right] .
\end{aligned}
$$

The vertices of the stability triangle $A B C$ in the $k_{1}, k_{2}$ plane are determined by the three pole placements:

$$
\left[\begin{array}{l}
\boldsymbol{k}_{A}^{T} \\
\boldsymbol{k}_{B}^{T} \\
\boldsymbol{k}_{C}^{T}
\end{array}\right]=\left[\begin{array}{rrr}
1 & 2 & 1 \\
-1 & 0 & 1 \\
1 & -2 & 1
\end{array}\right]\left[\begin{array}{rr}
5 & 6 \\
6 & 4 \\
4 & -8
\end{array}\right]=\left[\begin{array}{rr}
21 & 6 \\
-1 & -14 \\
-3 & -10
\end{array}\right] .
$$

Fig. 6 shows the stability triangle $A B C$. The circle with center $z=0.45$ and radius $r=0.5$ in the $z$-plane corresponds to a triangle $D E F$ with vertices

$$
\begin{aligned}
{\left[\begin{array}{c}
\boldsymbol{k}_{D}^{T} \\
\boldsymbol{k}_{E}^{T} \\
\boldsymbol{k}_{F}^{T}
\end{array}\right] } & =\left[\begin{array}{rrr}
0.0025 & 0.1 & 1 \\
-0.0475 & -0.9 & 1 \\
0.9025 & -1.9 & 1
\end{array}\right]\left[\begin{array}{rr}
5 & 6 \\
6 & 4 \\
4 & -8
\end{array}\right] \\
& =\left[\begin{array}{rr}
4.6125 & -7.585 \\
-1.6375 & -11.885 \\
-2.8875 & -10.185
\end{array}\right] .
\end{aligned}
$$

Only solutions in $D E F$ will be admissible in the following. Several aspects of design in the $\mathcal{K}$ space will now be illustrated by Example 2 .

\section{B. Actuator Constraints}

In many control systems actuator constraints of the type $|u(t)| \leqslant U$ for all $t$ have to be considered. One approach to treat this constraint indirectly is to avoid the saturation as far as possible by using small feedback gains, such that the system operates primarily in the linear range. For state feedback or static output feedback

$$
|u(t)|=\left|\boldsymbol{k}^{T} \boldsymbol{x}(t)\right| \leqslant\|\boldsymbol{k}\| \cdot\|\boldsymbol{x}(t)\|
$$

with equality for the worst case of $\boldsymbol{x}(t)$ (i.e., $\boldsymbol{x}=\boldsymbol{c} \boldsymbol{k}$ for some $c \neq 0$ ). Assuming that all state variables have been normalized to their maximum value, the norm $\|\boldsymbol{k}\|=\sqrt{\boldsymbol{k}^{\prime} \boldsymbol{k}}$, i.e., the distance from the origin in the $\mathcal{K}$ space can be used as a measure for $|u|$. This provides a 


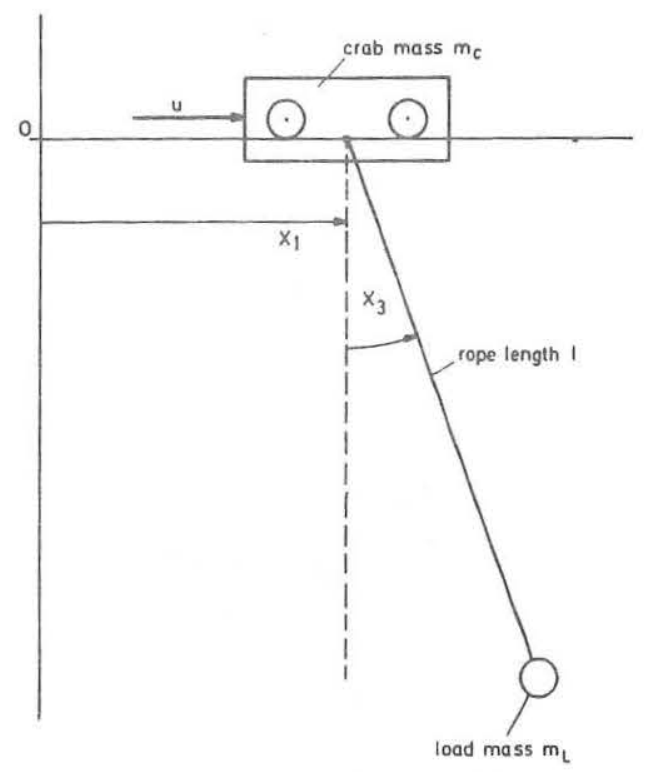

Fig. 5. A crane or loading bridge.

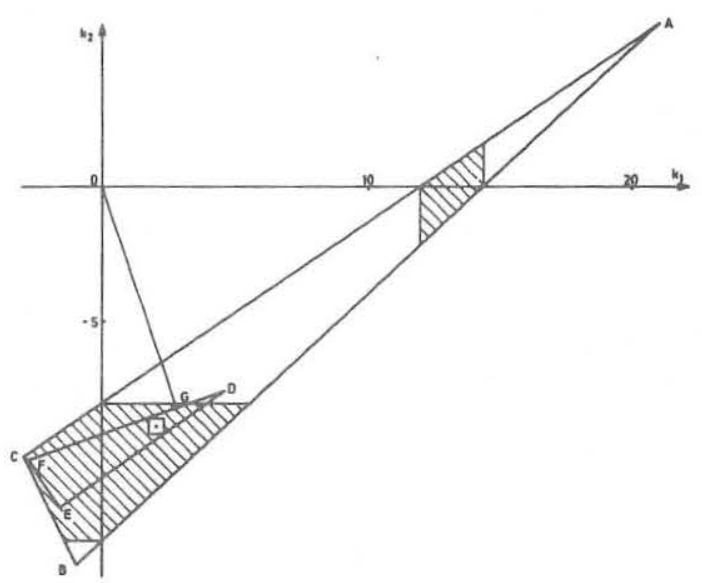

Fig. 6. Design in $\mathcal{K}$ plane for a second-order discrete-time system.

criterion for the selection of a gain from the admissible set: choose the point closest to the origin. In the example of Fig. 6 this is $G$ with $\boldsymbol{k}_{G}^{T}=[2.8-8.2]$. Equation (27) is here

$$
\boldsymbol{p}^{T}=\boldsymbol{k}^{T}\left[\begin{array}{rr}
-4 & 6 \\
6 & -5
\end{array}\right] \cdot \frac{1}{16}=\left[\begin{array}{ll}
4 & -4
\end{array}\right]
$$

Thus, $\boldsymbol{k}_{G}^{T}$ maps into $\boldsymbol{p}_{G}^{T}=\left[\begin{array}{ll}0.225 & -0.388\end{array}\right]$ with eigenvalues $z_{1,2}=0.194 \pm j 0.433$. This is the most gentle way to bring the double eigenvalue at $z=2$ into $\Gamma_{0.5}$. Note that the gain reduction margin of $G$ is 21 percent. The maximum gain reduction margin would be achieved at $E$ where it is 26 percent.

\section{Robustness with Respect to Measurement Noise and Quantization Effects in the Controller}

The feedback control law may be implemented approximately in a short wordlength microprocessor as

$$
u+\Delta_{1} u+\Delta_{2} u \approx\left(\boldsymbol{k}^{T}+\Delta \boldsymbol{k}^{T}\right)(x+\Delta x)
$$

where $\Delta_{2} u$ is the output quantization error, which may be neglected here as well as the product of small terms $\Delta \boldsymbol{k}^{T} \cdot \Delta x$. Then

$$
\Delta_{1} u \approx k^{T} \Delta x+\Delta k^{T} x
$$

is the error resulting from measurement noise and input quantization in $\Delta \boldsymbol{x}$ and quantization $\Delta \boldsymbol{k}^{T}$ of the feedback gains. The first term $\boldsymbol{k}^{T} \Delta x$ is kept small by minimizing $\|\boldsymbol{k}\|$ as illustrated in Section IV-B. The second term requires that some distance from the boundary must be kept as safety margin.

In the example of Fig. 6 let $\Delta \boldsymbol{k}^{T}=\left[\begin{array}{l} \pm 0.3 \pm 0.3 \\ \text {. This }\end{array}\right.$ margin is maintained at the center of the $0.6 \times 0.6$ square inside $D E F$ (indicated by omitted shading) which is moved as close as possible to $G$. It results in

$$
\begin{aligned}
\boldsymbol{k}^{T} & =\left[\begin{array}{ll}
2 & -8.9
\end{array}\right], \\
\boldsymbol{p}^{T} & =\left[\begin{array}{ll}
0.1625 & -0.46875
\end{array}\right], \\
z_{1,2} & =0.234 \pm j 0.328 .
\end{aligned}
$$

\section{Robustness with Respect to Sensor Failures}

Sensor failures are assumed to occur in the form that the sensor output is no longer correlated with the measured variable. As far as the characteristic equation is concerned, this is equivalent to having a sensor output zero. There may be a bias or other noise term introduced by the failed sensor. This noise term can be considered as an external input. This may require that the failure is detected and the failed sensor is removed from the control system. Then the control law may also be changed. However, for this latter decision there should be sufficient time to come to a reliable decision without false alarms. This requires that after the failure the system at least remains stable with some stability margin. In other applications it may suffice to be able to continue the mission after a sensor failure without removal of the failed sensor, e.g., to drive an automobile safely to a service station to get a broken sensor replaced, such that optimal fuel economy, emission control, acceleration, etc., is regained.

It is not reasonable to require the poles to remain in the same region $\Gamma$ as in the unfailed situation; thus, a relaxed "emergency region" $\Gamma_{E}$ is introduced. Here is the robustness problem: consider $M$ failures of a sensor or combinations of sensors leading to the crippled feedback vectors $\boldsymbol{k}_{m}^{T}, m=1,2, \cdots, M$ in which the appropriate elements of $\boldsymbol{k}^{T}$ are replaced by zero. Find $\boldsymbol{k}^{T}$ such that all zeros of

$$
\prod_{m=1}^{M} \operatorname{det}\left(\lambda \boldsymbol{I}-\boldsymbol{A}+\boldsymbol{b} \boldsymbol{k}_{m}^{T}\right)=0
$$

lie in the emergency region $\Gamma_{E}$ in the $\lambda$-plane. The emergency specification is robust with respect to a failure of sensor $i$ if and only if in the $\mathcal{K}$ space the projection of $\boldsymbol{k}^{T}$ into the subspace $k_{i}=0$ is in the emergency region $K_{\Gamma E}$.

Fig. 7 shows an example of emergency and nominal regions in the $k_{1}-k_{2}$-plane. If we choose $\boldsymbol{k}^{T}$ at point 1 , then the projection on the $k_{2}$ axis is inside the emergency boundary, i.e., $\Gamma_{E}$ is robust with respect to a failure of sensor 1. It is, however, not robust with respect to a failure of sensor 2 , since the projection on the $k_{1}$ axis is outside the emergency region. Points in the shaded region 


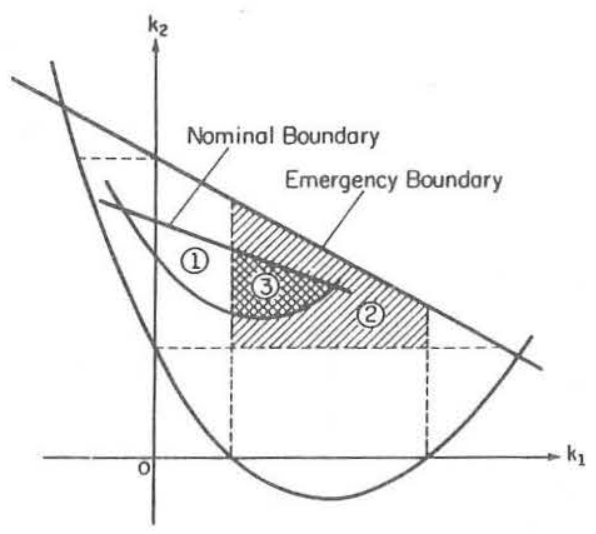

Fig. 7. Robustness with respect to sensor failures.

are robust with respect to failure of either sensor. For no $\boldsymbol{k}^{T} \Gamma_{E}$ is robust with respect to failures of sensors 1 and 2, since the origin $k_{1}=k_{2}=0$ lies outside the emergency region. Point 3 also meets the nominal specification and is a good candidate for further study and simulation. Since the nominal boundary intersects the $k_{2}$ axis, an alternative to the robust solution 3 is to eliminate the $x_{1}$ sensor and to multiplex the $x_{2}$ sensor. This would maintain the nominal specifications under a failure of one of the $x_{2}$ sensors. However, it requires failure detection with at least three $x_{2}$ sensors.

In the example of Fig. 6 it turns out that for the previously selected solution (41), stability is robust with respect to failure of sensor 1 since it is in the shaded region around the $k_{2}$-axis. However, no reasonable tradeoff can be made to achieve robustness with respect to failure of sensor 2, i.e., to go to the right shaded region. In other words, the sensor for $x_{2}$ is the more important one. If it is not reliable and the mission is critical, then this sensor must be multiplexed.

Independent of the chosen controller structure, a necessary condition for robustness of $\Gamma_{E}$ with respect to a failure of one or more sensors is that the eigenvalues of $A$ outside $\Gamma_{E}$ remain observable after a failure. It is an open question whether this condition is also sufficient, i.e., whether there exists a controller structure and constant parameters in it such that robustness of $\Gamma_{E}$ with respect to all failure combinations which leave the eigenvalues outside $\Gamma_{E}$ observable is achieved.

\section{E. Robustness with Respect to Large Plant Parameter Variations}

Assume that the plant model

$$
\dot{x}=A(\theta) x+b(\theta) u
$$

is given for several typical values of the physical parameter vector $\boldsymbol{\theta}$, i.e., $\boldsymbol{A}_{j}=\boldsymbol{A}\left(\boldsymbol{\theta}_{j}\right), \boldsymbol{b}_{j}=\boldsymbol{b}\left(\theta_{j}\right), j=1,2 \cdots J$. A fixedstate feedback is sought, such that all zeros of

$$
\operatorname{det}\left(\lambda I-A_{j}+b_{j} k^{T}\right)=0
$$

are located in a specified region $\Gamma$ in the $\lambda$-plane, i.e., $p$ is located in the corresponding region $P_{\Gamma}$ in the $\mathcal{P}$ space.

Each pair $A_{j}, b_{j}$ leads to a different matrix $\boldsymbol{E}_{j}$ in (20). $P_{\Gamma}$ is mapped by $\boldsymbol{k}^{T}=\left[\begin{array}{ll}\boldsymbol{p}^{T} & 1\end{array}\right] \boldsymbol{E}_{j}$ into $J$ different regions $K_{\Gamma j}$ in the $\mathcal{K}$ space. The set of solutions to the problem (44), if it exists, is the intersection of these $J$ regions in the $\mathscr{K}$ space. If no intersection for all $j=1,2 \cdots J$ exists, then it can be tested whether at least a group of plant models can be nicely stabilized with one gain and it may be necessary to switch to a different gain for a different group of plant models.

As an example let $n=2, J=2$, and $\Gamma$ be circular. The solution is the intersection of two triangles. This intersection may be empty, a triangle, a quadrangle, a pentagon, or a hexagon. With an increasing number $J$ of plant models, many more cases of polygons have to be distinguished. It is not advisable to solve this problem numerically, i.e., to calculate the vertices of the intersecting polygon, since the designer is not interested in the number of vertices. For him it is important to know whether the intersection is nonempty and what its approximate size and extension is. In the interaction between a designer and a computer-aided design system this task of finding the intersection on a display should be assigned to the designer.

Now let $n=3, J=2$, and $\Gamma$ be circular. The nice stability regions $K_{\Gamma j}$ are affine to the region of Fig. 4. For each operating point $j$ they are determined by four pole placements of the vertices $A, B, C$, and $D$. The complex boundary is then constructed by dividing each edge into equal segments and connecting the corresponding points by straight lines.

Here we can draw an important conclusion from the possible intersections of two bodies of the shape of Fig. 4: the admissible set of solutions may be disconnected. This is seen if we visualize a second body of the same shape upside down such that two intersections of the tips near $A$ and $D$ occur. In numerical methods a systematic search may be necessary in order to find all parts of the admissible set. Here it is helpful to limit this search to the intersection of the convex hulls, in the example the two tetrahedra. If this intersection is empty, then no robust solution exists. If it is nonempty, then this intersecting polyhedron defines the search space, where at least the necessary condition for a robust solution is satisfied.

For arbitrary $n$ a point $\boldsymbol{k}$ in the $\mathcal{K}$ space can be tested algebraically as to whether it belongs to the simplex of stability or nice stability. This is formulated as Theorem 2.

Theorem 2: A necessary condition for $\boldsymbol{k}^{T}$ to stabilize $\boldsymbol{x}(k+1)=\left(\boldsymbol{A}-\boldsymbol{b} \boldsymbol{k}^{T}\right) \boldsymbol{x}(k)$ is that all elements of

$$
\boldsymbol{\mu}^{T}=\left[\begin{array}{ll}
\boldsymbol{k}^{T} & 1
\end{array}\right]\left[\begin{array}{cc}
\boldsymbol{k}_{0}^{T} & 1 \\
\boldsymbol{k}_{1}^{T} & 1 \\
\vdots & \vdots \\
\boldsymbol{k}_{n}^{T} & 1
\end{array}\right]^{-1}
$$


are positive, where $\boldsymbol{k}_{i}^{T}$ assigns the characteristic polynomial $P(z)=(z-1)^{n-i}(z+1)^{i}, i=0,1,2 \cdots n$.

Proof: Substitute (15) into (20):

$$
\boldsymbol{k}^{T}=\left[\begin{array}{ll}
\boldsymbol{p}^{T} & 1
\end{array}\right] \boldsymbol{E}=\boldsymbol{\mu}^{T}\left[\begin{array}{cc}
\boldsymbol{p}_{0}^{T} & 1 \\
\boldsymbol{p}_{1}^{T} & 1 \\
\vdots & \vdots \\
\boldsymbol{p}_{n}^{T} & 1
\end{array}\right] \boldsymbol{E}=\boldsymbol{\mu}^{T}\left[\begin{array}{c}
\boldsymbol{k}_{0}^{T} \\
\boldsymbol{k}_{1}^{T} \\
\vdots \\
\boldsymbol{k}_{n}^{T}
\end{array}\right]
$$

This is augmented by (14):

$$
\left[\begin{array}{ll}
\boldsymbol{k}^{T} & 1
\end{array}\right]=\boldsymbol{\mu}^{T}\left[\begin{array}{cc}
\boldsymbol{k}_{0}^{T} & 1 \\
\boldsymbol{k}_{1}^{T} & 1 \\
\vdots & \vdots \\
\boldsymbol{k}_{n}^{T} & 1
\end{array}\right]
$$

and inverted to give (45).

Q.E.D.

The generalization to other circles is obvious. Different plant models result in different $\boldsymbol{E}_{j}$ and thus different vertices $\boldsymbol{k}_{o}^{T} \cdots \boldsymbol{k}_{n}^{T}$ of the simplex and different barycentric coordinates $\boldsymbol{\mu}_{j}^{T}$ in (45). $\boldsymbol{k}^{T}$ is in the intersection of simplexes if all these coordinates are positive. This is a necessary condition for a robust feedback.

For different values $\boldsymbol{\theta}_{j}$ of the physical parameter vector, different regions $\Gamma_{j}$ in the $\lambda$ plane may be given and the intersections of the corresponding $\mathscr{K}$ space regions must be found. Such parameter-dependent pole regions are given in military specifications for aircraft modes [6]. This reflects the fact that the pilot, for example, expects the aircraft to react more slowly in landing approach than in terrain following at high speeds. A general recommendation for the design of robust control systems with actuator constraints is to try not to make a slow plant fast or a fast plant slow by feedback. This is an essential difference to all high-gain design concepts which locally reduce the sensitivity of a nominal trajectory $\boldsymbol{x}(t)$ or deviation from a reference model response under plant parameter variations. Pole region assignment in connection with soft feedback, i.e., minimum $\|\boldsymbol{k}\|$, offers more flexibility to also accommodate large parameter variations with medium control magnitudes. This point is further illustrated by the crane example (32). The gain scheduling control of (35) keeps the eigenvalues constant in spite of load variations. But even if $m_{L}$ is known, this control is undesirable because in view of constraints on the available force $|u|$, positioning of the empty hook should be performed faster than positioning of the maximum load. It is important to also keep this point in mind in multiinput problems where this aspect may be obscured by the large number of remaining free parameters after the poles have been assigned. Even the best choice of these parameters cannot compensate for speeding up the modes too much by pole placement.

\section{Partial Gain Assignment, OUtput Feedback}

The concept of graphical $\mathcal{K}$ space design of robust control systems was introduced for a second-order system with only two free feedback gains $k_{1}$ and $k_{2}$. Also threedimensional regions and their intersections can be made visible by computer graphic methods which permit animation, rotation, change of viewing direction and distance, multicolor, and stereoscopic displays.

A fourth dimension of the parameter space can be translated into time, but here we would reach the limits of our imagination. The design can then be performed in iterative steps, such that in each step only the influence of two or three parameters is studied, while all other gains are fixed. Also other design constraints, like output feedback or sensor failure cases, can require that some gains in $\boldsymbol{k}^{T}$ are fixed in advance. This means that we are looking for a solution in a subspace of $\mathscr{K}$. Such a solution may not exist; take for example Fig. 6 and fix $k_{1}$ to be bigger than $k_{1}(A)$. Then there does not exist a stabilizing $k_{2}$. The set of admissible solutions may also become disconnected, even if it is connected in the $\mathcal{K}$ space; take, for example, a stability region such as that in Fig. 4 , map it into the $\mathscr{K}$ space, and fix one $k_{i}$ such that the plane $k_{i}=c$ intersects the two tips of the stability region.

Example 3 (Disconnected Stability Regions in a Subspace of $\mathscr{T})$ :

$$
x(k+1)=\left[\begin{array}{lrl}
0 & 1 & 0 \\
0 & 0 & 1 \\
0.6 & -2 & 2.1
\end{array}\right] x(k)+\left[\begin{array}{l}
0 \\
0 \\
1
\end{array}\right] u(k) .
$$

The system is open-loop unstable (eigenvalues $z_{1}=0.5$, $z_{2,3}=0.8 \pm j \sqrt{0.56}$ ). Fix $k_{2}=0$ (output feedback) and find the set of stabilizing gains in the $k_{1}-k_{3}$-plane. The real root boundaries are the straight lines

$$
\begin{array}{ll}
\text { for } z=1 & k_{3+}=-k_{1}-0.3 \\
\text { for } z=-1 & k_{3-}=-k_{1}+5.7
\end{array}
$$

and the complex root boundary is the hyperbola

$$
k_{3 c}=k_{1}+1.5+1 /\left(k_{1}-0.6\right) .
$$

Fig. 8 shows the three boundaries and the two disconnected stabilizing regions. Its vertices are

\begin{tabular}{rrr} 
& \multicolumn{1}{c}{$k_{1}$} & \multicolumn{1}{c}{$k_{3}$} \\
$E$ & -0.4 & 0.1 \\
$F$ & 0.1 & -0.4 \\
$G$ & 1.1 & 4.6 \\
$H$ & 1.6 & 4.1.
\end{tabular}

Nonconvex and disconnected solution sets such as in this example lead to difficulties in numerical algorithms. Sirisena and Choi [7] formulate the problem of placing poles in a specified region by output feedback as minimization of a function $J$ which becomes zero if a solution is found. Their conclusion from computational experience is, "If, however, a local (nonzero) minimum of $J$ is 
reached, the algorithm should be restarted with a different initial value of the feedback matrix. Repeated failure to reduce $J$ to zero would indicate the absence of a solution."

An alternative is a search in the region where the necessary condition to be in the simplex is satisfied. In Fig. 8 this is the quadrangle $E F H G$; in general, it is the polyhedral cross section of the subspace given by the fixed $k_{i}$ with the simplex. If no such cross section exists, it can be concluded that no solution exists. The example indicates that points near the real root boundaries are promising candidates.

The effect of fixed gains on the characteristic polynomial can be seen by (20):

$$
\begin{aligned}
\boldsymbol{k}^{T} & =\left[\begin{array}{llll}
k_{1} & k_{2} & \cdots & k_{n}
\end{array}\right]=\left[\begin{array}{ll}
\boldsymbol{p}^{T} & 1
\end{array}\right] \boldsymbol{E}, \\
\boldsymbol{E} & =\left[\begin{array}{llll}
\boldsymbol{\eta}_{1} & \boldsymbol{\eta}_{2} & \cdots & \eta_{n}
\end{array}\right] .
\end{aligned}
$$

Fixing $k_{i}$ implies a linear relationship

$$
k_{i}=\left[\begin{array}{ll}
p^{T} & 1
\end{array}\right] \boldsymbol{\eta}_{i}
$$

between the coefficients of the characteristic polynomial. If $g$ gains are fixed it is convenient to factor out a $g$ th-order polynomial $R(\lambda)$ from $P(\lambda)=R(\lambda) Q(\lambda)$ such that assigning a remainder polynomial $Q(\lambda)$ together with the $g$ gains determines $R(\lambda)$ and the $n-g$ free gains of $\boldsymbol{k}^{T}$. The coefficients of the product polynomial may be written

$$
\begin{aligned}
& {\left[\begin{array}{ll}
\boldsymbol{p}^{T} & 1
\end{array}\right]=\left[\begin{array}{lll}
p_{0} & \cdots & p_{n-1} \\
1
\end{array}\right]} \\
& {\left[\begin{array}{ll}
p^{T} & 1
\end{array}\right]=\left[\begin{array}{ll}
r^{T} & 1
\end{array}\right]\left[\begin{array}{c}
S \\
t^{T}
\end{array}\right]} \\
& \boldsymbol{k}^{T}=\left[\begin{array}{ll}
\boldsymbol{k}_{a}^{T} & \boldsymbol{k}_{b}^{T}
\end{array}\right]=\left[\begin{array}{ll}
\boldsymbol{p}^{T} & 1
\end{array}\right]\left[\begin{array}{ll}
\boldsymbol{E}_{a} & \boldsymbol{E}_{b}
\end{array}\right] \\
& =\left[\begin{array}{ll}
r^{T} & 1
\end{array}\right]\left[\begin{array}{c}
S \\
t^{T}
\end{array}\right]\left[\begin{array}{ll}
E_{a} & E_{b}
\end{array}\right] \\
& k_{b}^{T}=r^{T} S E_{b}+t^{T} E_{b}
\end{aligned}
$$

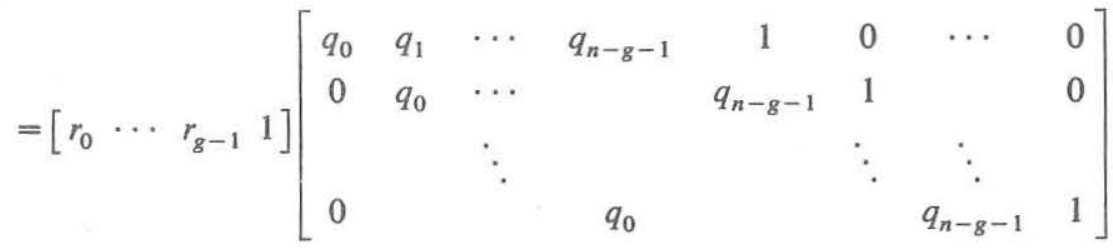

where $S$ is a $g \times(n+1)$ matrix and $\boldsymbol{t}^{T}$ a $1 \times(n+1)$ vector. Let $\boldsymbol{k}_{b}^{T}$ be the fixed gains, which for convenience are chosen to be the last $g$ gains in $\boldsymbol{k}^{T}$. Then

which can be solved for

$$
\boldsymbol{r}^{T}=\left(\boldsymbol{k}_{b}^{T}-\boldsymbol{t}^{T} E_{b}\right)\left(S E_{b}\right)^{-1}
$$

if the $g \times g$ matrix $S E_{b}$ is invertible. Note that this condition does not depend on the values of $\boldsymbol{k}_{b}^{T}$; thus, this is the same problem as in output feedback $\boldsymbol{k}_{b}^{T}=\mathbf{0}$ where certain pole locations cannot be achieved. Variable $\boldsymbol{k}_{a}^{T}$ is determined by

$$
\boldsymbol{k}_{a}^{T}=\left[\begin{array}{ll}
\boldsymbol{p}^{T} & 1
\end{array}\right] \boldsymbol{E}_{a}=\left(\boldsymbol{r}^{T} \boldsymbol{S}+\boldsymbol{t}^{T}\right) \boldsymbol{E}_{a} .
$$

Assigning the $n-g$ eigenvalues of $Q(\lambda)$ determines $S$. The remaining $g$ eigenvalues can be determined by factoring the residual polynomial $R(\lambda)$ with coefficients given by (50).

Example 4 (Fixed Feedback Gains): For the crane of Example 2 let $k_{1}$ and $k_{4}$ be fixed, i.e., $\boldsymbol{k}_{a}^{T}=\left[\begin{array}{ll}k_{2} & k_{3}\end{array}\right], \boldsymbol{k}_{b}^{T}=$ $\left[\begin{array}{ll}k_{1} & k_{4}\end{array}\right]$ :

$\boldsymbol{E}_{a}=\left[\begin{array}{cc}0 & l^{2} m_{c} / g \\ l m_{c} / g & 0 \\ 0 & -l m_{c} \\ 0 & 0 \\ 0 & \left(m_{c}+m_{L}\right) g\end{array}\right] \quad \boldsymbol{E}_{b}=\left[\begin{array}{cc}l m_{c} / g & 0 \\ 0 & l^{2} m_{c} / g \\ 0 & 0 \\ 0 & -l m_{c} \\ 0 & 0\end{array}\right]$.

Then by (50)

$$
\begin{aligned}
r^{T} & =\left[\begin{array}{ll}
r_{0} & r_{1}
\end{array}\right] \\
& =\left[\begin{array}{ll}
k_{1} & k_{4}+q_{1} l m_{c}
\end{array}\right] \cdot\left[\begin{array}{cc}
q_{0} l m_{c} / g & q_{1} l^{2} m_{c} / g \\
0 & \operatorname{lm} m_{c}\left(q_{0} l / g-1\right)
\end{array}\right]^{-1} .
\end{aligned}
$$

The inverse exists if $q_{o} \neq g / l$ and $q_{o} \neq 0$. Then

$$
r_{0}=\frac{k_{1} g}{q_{0} l m_{c}}, \quad r_{1}=\frac{q_{1} m_{c}-k_{1} q_{1} / q_{0}+k_{4} / l}{m_{c}\left(q_{0} l / g-1\right)}
$$

and with (51)

$$
\begin{aligned}
k_{2}\left(q_{0}, q_{1}, k_{1}, k_{4}\right) & =\frac{q_{0} q_{1} m_{c} l-k_{1} g q_{1} / q_{0}+k_{4} q_{0}}{q_{0} l-g} \\
k_{3}\left(q_{0}, q_{1}, k_{1}, k_{4}\right) & =l\left(k_{1} / q_{0}-m_{c}\right)\left(q_{0}+\frac{q_{1}^{2} g}{q_{0} l-g}\right) \\
& +g\left(\frac{k_{4} q_{1}}{q_{0} l-g}-\frac{k_{1}}{q_{0}}+m_{c}+m_{L}\right) .
\end{aligned}
$$

$k_{1}$ will be fixed by the following consideration. Assume a force limitation $|u(t)|<U$ for all $t$ for a typical operation of the crane, i.e., a displacement of a load at rest, $x(0)=$ $\left[\begin{array}{llll}L & 0 & 0 & 0\end{array}\right]^{T}, L>0$ (e.g., length of a loading bridge) to a final position $\boldsymbol{x}\left(t_{E}\right)=\left[\begin{array}{llll}0 & 0 & 0 & 0\end{array}\right]^{T}$. Typical responses of sufficiently stabilized cranes show an initial peak $u(0)$ of the force as the maximum value of $|u(t)|$. A simple approach to avoid saturation is, therefore, to meet a necessary condition by fixing $|u(0)|=U$ and checking the conditions for $\dot{u}(0) / u(0)<0$. Then $|u(t)|$ for $t>0$ may be checked in a simulation. Here

$$
u(0)=-\boldsymbol{k}^{T} x(0)=-k_{1} L
$$




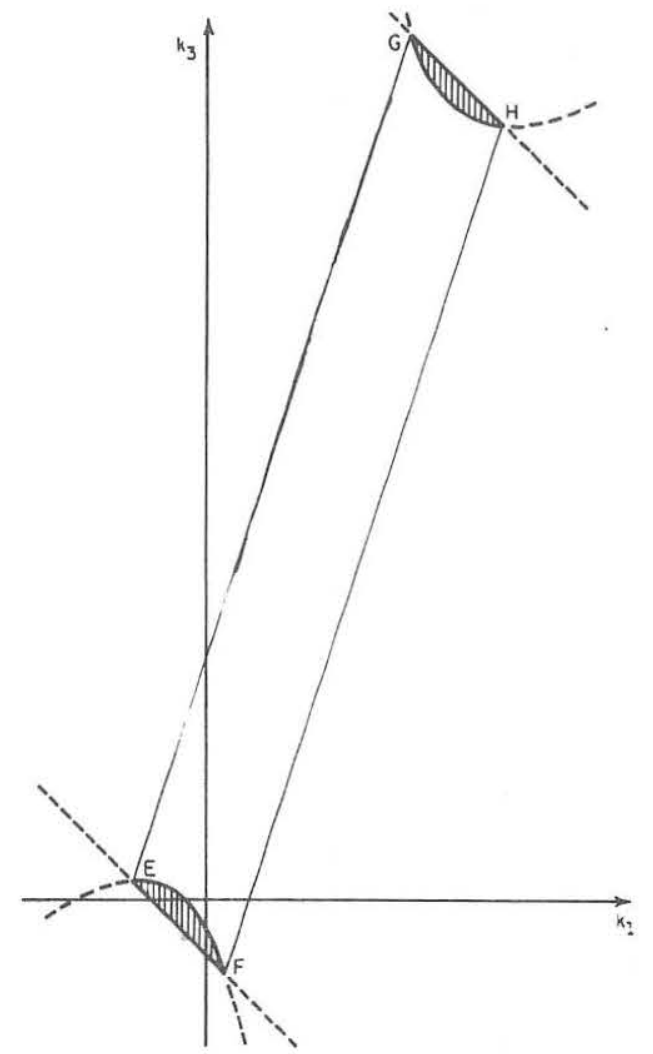

Fig. 8. Disconnected stability region in $k_{1}-k_{3}$ subspace.

$$
\begin{aligned}
\dot{u}(0) & =-\boldsymbol{k}^{T}\left(\boldsymbol{A}-\boldsymbol{b} \boldsymbol{k}^{T}\right) \boldsymbol{x}(0) \\
& =L k_{1}\left(k_{2}-k_{4} / l\right) / m_{c}=L k_{1} p_{3} .
\end{aligned}
$$

Thus, $\dot{u}(0) / u(0)=-1 / p_{3} \leqslant 0$ for all stabilizing feedbacks and $|u(0)|=U$ results in $k_{1}=U / L$.

It is desirable to avoid the difficult measurement of the rope angular velocity $x_{4}$. Thus, $k_{4}=0$ is chosen. Then by (52)

$$
r_{0}=\frac{U g}{L m_{c} q_{0}}, \quad r_{1}=\frac{q_{1} g\left(1-U / L m_{c} q_{0}\right)}{l q_{0}-g} .
$$

Variables $r_{o}$ and $r_{1}$ are the coefficients of the residual polynomial which is obtained after $q_{o}$ and $q_{1}$ have also been fixed. Necessary and sufficient conditions for stability are $q_{o}>0, q_{1}>0, r_{o}>0, r_{1}>0$. With (53)

$$
\begin{aligned}
k_{2}\left(q_{0}, q_{1}\right)= & \frac{q_{0} q_{1} m_{c} l-U g q_{1} / L q_{0}}{q_{0} l-g} \\
k_{3}\left(q_{0}, q_{1}\right)= & -\operatorname{lm}_{c}\left(q_{0}+\frac{q_{1}^{2} g}{q_{0} l-g}\right) \\
& +\frac{U}{L q_{0}}\left(q_{0} l-g+\frac{l q_{1}^{2} g}{q_{0} l-g}\right)+\left(m_{c}+m_{L}\right) g .
\end{aligned}
$$

For given values of $m_{c}, m_{L}, l, g, U$, and $L$ we can now use (52) to map points on a boundary of $\Gamma$ in the eigenvalue plane, i.e., $q_{o}-q_{1}$ pairs, into the $k_{2}-k_{3}$-plane. If one
TABLE I

\begin{tabular}{cccl}
\hline \hline & $k_{2}$ & $k_{30}$ & Eigenvalues \\
\hline$A$ & 4233 & -84292 & $s_{1,2}=-0.25, s_{3,4}=-1.867 \pm j 2.125$ \\
$B$ & 2367 & -35012 & $s_{1,2}=-0.275 \pm j 0.231, s_{3,4}=-0.908 \pm j 1.746$ \\
$C$ & 2769 & -22056 & $s_{1}=-0.25, s_{2}=-1.337, s_{3,4}=-0.591 \pm j 1.071$ \\
\hline
\end{tabular}

of the resulting regions corresponds to the case that all eigenvalues are inside $\Gamma$, then this is the admissible set of solutions. Several such sets may be plotted, e.g., for different load masses $m_{L}$ or rope lengths $l$ in order to find a robust solution in the intersection. In order to illustrate this, $m_{L}$ is assumed unknown and numerical values for the other parameters are given as $m_{c}=1000 \mathrm{~kg}, l=10 \mathrm{~m}$, $g=10 \mathrm{~m} / \mathrm{s}^{2}, U=5000 \mathrm{~N}$, and $L=10 \mathrm{~m}$.

Example 1 showed that only $k_{3}=k_{30}=m_{L} g$ depends on the load mass $m_{L} \cdot k_{30}=k_{3}-10 m_{L}$ is determined first. For $k_{1}=U / L=500$ and $k_{4}=0$ find the region in the $k_{2}-k_{30^{-}}$ plane for which all eigenvalues are left of the hyperbola

$$
\omega^{2}=(2 \sigma)^{2}-1 / 2^{2}
$$

in the $s$-plane. Then for the complex root boundary from (10)

$$
q_{0}=5 \sigma^{2}-0.25, \quad q_{1}=-2 \sigma
$$

and by (55) and (56)

$$
\begin{aligned}
r_{0} & =1 / 2 q_{0}, \quad r_{1}=\frac{q_{1}\left(1-1 / 2 q_{0}\right)}{q_{0}-1} \\
k_{2 c} & =\frac{1000 q_{1}}{q_{0}-1}\left(q_{0}-1 / 2 q_{0}\right) \\
k_{30 c} & =10000\left(1 / 2 q_{0}-1\right)\left[q_{0}-1+q_{1}^{2} /\left(q_{0}-1\right)\right] \\
k_{3 c} & =k_{30 c}+10 m_{L} .
\end{aligned}
$$

The nice stability region will be constructed in the $k_{2}-k_{30}$-plane. The complex root boundary $k_{2}(\sigma), k_{30}(\sigma)$ is obtained by substituting values $\sigma<-0.25$ into (58) and $q_{o}$ and $q_{1}$ into (60) and (61). The real root boundary at $\sigma_{R}=-0.25$ follows from (8) with $k_{1}=500, k_{4}=0$ as the straight line

$$
k_{3 R}=k_{30 R}+10 m_{L}, \quad k_{30 R}=95625-42.5 K_{2} .
$$

Both boundaries are shown in Fig. 9. For $\sigma=-0.25$ the complex root boundary starts at point $A$. With increasing $\sigma$ it goes through point $B$ and for $\sigma \rightarrow-0.5$, i.e., $q_{o} \rightarrow 1$ to infinity. In general, this singularity occurs at $q_{o}=g / l$. For $\sigma<-0.5$ the complex root boundary returns from the opposite side to intersect the real root boundary at $C$ and itself at $B$.

Note that the characteristic polynomial is obtained by (58) and (59) in factorized form. Thus, the determination of the eigenvalues is easy. They are given together with the $k_{2}$ and $k_{30}$ coordinates in Table I.

At $A$ the real and complex root boundaries intersect, i.e., there is a double pole at $s_{1,2}=-0.25$. At $B$ the 


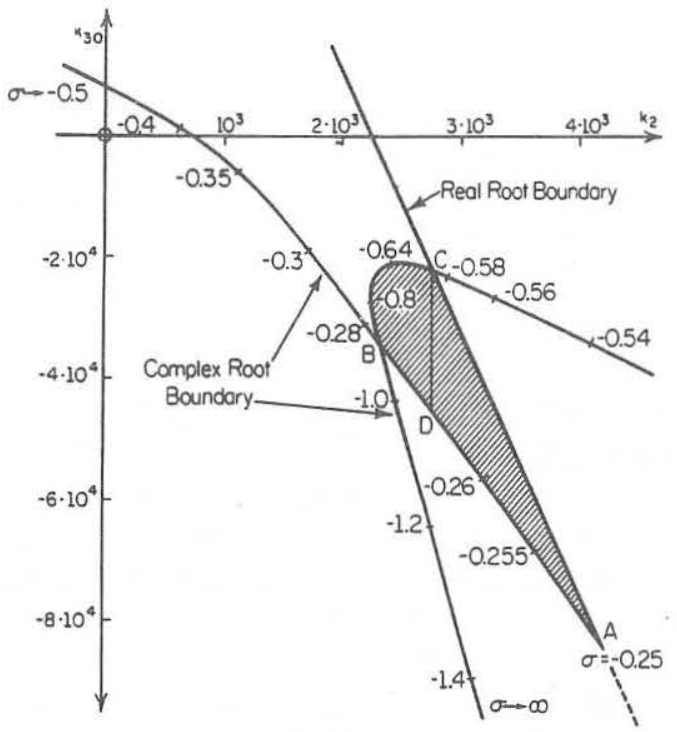

Fig. 9. Nice stability region for crane with $k_{1}=500, k_{4}=0$.

complex boundary intersects itself. Here we have two complex pairs of eigenvalues crossing the boundary simultaneously. At $C$ a real root at $s=-0.25$ crosses simultaneously with the complex pair $s_{3,4}$. The shaded region with vertices $A, B$, and $C$ corresponds to eigenvalues to the left of the hyperbola in $s$ plane.

It is easy now to find the fixed gain controller which accommodates the largest load variation.

The load mass enters only into $k_{3}=k_{30}+10 m_{L}$. In Fig. 9 the origin of the $k_{3}$-axis is identical to $k_{30}=0$ for $m_{L}=0$. With increasing load mass the shape of the region of nice stability is unchanged, but it is moved upwards by $10 m_{L}$ in the $k_{2}-k_{3}$-plane, or equivalently the origin of the $k_{3}$-axis is moved downwards by $10 m_{L}$ in the $k_{2}-k_{30}$ plane. Thus, for load variations of cranes it is not necessary to plot the shifted diagrams in order to find the intersection. The largest load variation can be accommodated at the largest extension of the nice stability region in $k_{3}$ direction. This is between $C$ and $D . D$ has the coordinates $k_{2}=2769, k_{30}=-45503$ and corresponds to the eigenvalues $s_{1,2}=-0.267 \pm j 0.680$ and $s_{3,4}=1.118 \pm j 1.872$. Thus, $k_{2}$ is chosen as 2769 . This results in an admissible load variation $m_{L}=(-22056+45503) / 10=2344.7 \approx 2345$ $\mathrm{kg}$. Assume that the weight of the empty hook is $50 \mathrm{~kg}$, then $k_{3}=-21556$ puts the eigenvalues for $m_{L}=50 \mathrm{~kg}$ at $s_{1}=-0.25, s_{2}=-1.337, s_{3,4}=-0.591 \pm j 1.071$ where $s_{1}$ and $s_{3,4}$ are on the boundary $\Gamma$. For $m_{L}=2395 \mathrm{~kg}$ the eigenvalues are at $s_{1,2}=-0.267 \pm j 0.680$ and $s_{3,4}=1.118 \pm$ $j 1.872$ where $s_{1,2}$ is on the boundary. In summary, the solution

$$
\boldsymbol{k}^{T}=\left[\begin{array}{llll}
500 & 2769 & -21556 & 0
\end{array}\right]
$$

gives the following properties of the control system.

1) Initial peak in the force $u$ limited to $500 L$ where $L$ is the required load displacement.

2) No measurement or estimation of the rope angular velocity $x_{4}$ is required.

3) Under the constraints 1) and 2) maximum possible load variation. The eigenvalues are left of $\omega^{2}=(2 \sigma)^{2}-1 / 2^{2}$ if and only if $50 \mathrm{~kg}<m_{L}<2395 \mathrm{~kg}$.

Now assume that the crane is designed for a maximum load of $3500 \mathrm{~kg}$, i.e., a gain scheduling is necessary. The second load range may be chosen as $1155 \mathrm{~kg}<m_{L}<3500$ $\mathrm{kg}$, and $k_{3}=-10506$. Then for $50 \mathrm{~kg}<m_{L}<1155 \mathrm{~kg}$, $k_{3}=-21556$ must be used and for $2395 \mathrm{~kg}<m_{L}<3500$ $\mathrm{kg}, k_{3}=-10506$. For the overlapping range $1155 \mathrm{~kg}<m_{L}$ $<2395 \mathrm{~kg}$ either gain is good, such that the crane operator can switch between high and low load based on his very crude load estimate which may be \pm 35 percent wrong. This wide overlap provides robustness of the gain scheduling scheme.

If the rope length of the crane is varied, the shape of the nice stability region in Fig. 6 changes and an intersection of various regions must be found.

All calculations were done by pocket calculator. The example shows that realistic problems of robust control system design can already be solved with this new method. Another application example was given by Franklin [8]. He designed a robust stabilization for the short period longitudinal mode of an F 4-E aircraft with canards. Uncontrolled, it is unstable in subsonic flight and unsufficiently damped in supersonic flight. Including the actuator the model is third-order with two outputs (accelerometer and gyro). For different altitudes and Mach numbers the eigenvalues were placed simultaneously in the regions given by military specifications. Also the short period mode had to remain separated from other modes and the bandwidth was limited in order to avoid the structural vibration frequency range.

Further design examples for a dc motor control and a sampled data control of the crane were given in [9].

\section{Multinnput and Dynamic Output Feedback PRoblems}

$$
\text { For a system with } p \text { inputs, } \boldsymbol{u}=\left[u_{1} \cdots u_{p}\right]^{T} \text {, }
$$$$
\dot{x}=A x+B u \text {. }
$$

The feedback matrix $u=-\boldsymbol{K}^{T} \boldsymbol{x}$ has $p \times n$ free parameters, which define a parameter space $\mathcal{K}$ of this dimension.

In the single-input case it was convenient to study the shape of parameter regions in principle in a canonical parameter space of the same dimension as $\mathscr{K}$. The particular plant then determined an affine mapping of this region into $\mathscr{K}$ space. In the same spirit it is possible to define a $p \times n$-dimensional canonical parameter space $\mathscr{P}_{c}[10]$. Its coordinates are elements of a $p \times n$ matrix $\boldsymbol{P}^{T}$, the generalization of $\boldsymbol{p}^{T}$ in (20). Also $\boldsymbol{E}$ in this equation has a generalization to the multiinput case and the result is a mapping from $\mathscr{P}_{c}$ to $\mathscr{K}$ which is basically of the form

$$
\boldsymbol{K}^{T}=\boldsymbol{M}\left[\boldsymbol{P}^{T} \boldsymbol{I}\right] \boldsymbol{E}
$$

where $\boldsymbol{M}$ is a nonsingular triangular matrix determined by $\boldsymbol{A}, \boldsymbol{B}$. The characteristic polynomial is then the determi- 
nant of a characteristic polynomial matrix with coefficients given by $\boldsymbol{P}^{T}$. More details are given in [9] and [10]. The dynamic output feedback problem can also be formulated as a multiinput problem for an augmented system.

If the parameter space methods show that for a given - controller structure no solution exists, then one of the possibilities is to assume a richer controller structure, dynamic output feedback of order $m$. The plant is augmented by $m$ controller states in $\boldsymbol{x}_{c}$ :

$$
\left[\begin{array}{l}
\dot{x} \\
\dot{x}_{c}
\end{array}\right]=\left[\begin{array}{cc}
A & 0 \\
0 & 0
\end{array}\right]\left[\begin{array}{l}
x \\
x_{c}
\end{array}\right]+\left[\begin{array}{ll}
B & 0 \\
0 & I
\end{array}\right]\left[\begin{array}{l}
u \\
u_{c}
\end{array}\right]
$$

and a state feedback

$$
\left[\begin{array}{c}
u \\
u_{c}
\end{array}\right]=-\left[\begin{array}{cc}
K & L \\
M & N
\end{array}\right]\left[\begin{array}{l}
x \\
x_{c}
\end{array}\right]
$$

with $m$ controller inputs in $\boldsymbol{u}_{c}$ is determined, which nicely stabilizes the system (66). The controller $\boldsymbol{M}, \boldsymbol{N}, \boldsymbol{L}$ with $n$ inputs and $p$ outputs may be written in a canonical basis, such that the feedback matrix in (67) contains $p n+m n+m p$ design parameters $[(p+m) \times(n+m)$ coefficients in (67) of which $m^{2}$ are normalized by the choice of an $m \times m$ transformation matrix].

For $\boldsymbol{A}$ in sensor coordinates and output feedback the columns of $\boldsymbol{K}$ and $\boldsymbol{M}$ corresponding to missing sensors are zero, and thus the number of free parameters is reduced by $p+m$ for each nonmeasured state or failed sensor. Thus, for $q$ measurements the controller has $(p+m) q+m p$ free design parameters. An actuator failure changes $n+m$ coefficients in a row of $\boldsymbol{K}$ and $\boldsymbol{L}$ to zero.

Although basically the generalization of parameterspace methods to multiinput dynamic output feedback problems is possible, the apparent difficulty is owing to the large number of design parameters. In practical design a more pragmatic way of finding feedback dynamics is more promising. An example was given in the already mentioned design of an aircraft control system [8]. As a result of the first design step robustness with respect to large plant parameter variations was achieved and it was shown that, with static output feedback, it is impossible to make the emergency specifications (damping 0.15 and minimum value for the natural frequency) robust with respect to a failure of the gyro or the accelerometer. Dynamic feedback was designed in the form of two filters connected to the gyro and accelerometer outputs, which provided a second independent signal for the case of failure of one sensor. This defined a four-dimensional parameter space of the feedback gains of the two sensors and their filters.

Finally a solution with two paralleled gyros and one accelerometer was found, which meets the nominal specifications for the unfailed system and after a failure of any single sensor, and meets the emergency specifications after failure of any two sensors, where these properties pertain to four very different flight conditions.

\section{CONCLUSIONS}

Problems which are usually approached by adaptive control and redundant components with failure detection may also have a fixed gain robust solution. In many cases such a simple robust controller will be sufficient, as was shown for examples of a crane and an aircraft. Also, if adaptive control and failure detection is used in order to improve the performance, it is helpful to have sufficient time for reliable and accurate identification, adaptation, and failure detection without false alarms. This time can be provided by a robust stabilization system which takes care that immediately after a component failure or sudden large parameter change nothing very bad happens. In other words, the adaptation and failure detection is no longer vital for stabilization.

Essential for the design of robust controllers are that only physically reasonable requirements are made, which lead to small feedback gains and control inputs. When the plant is slow it remains slow; when it is fast it remains fast. After component failures, only reduced performance given by emergency specifications can be achieved.

This becomes possible if the pole assignment requirement is relaxed to a pole region assignment. It was shown how such a pole region is represented as a region in the parameter space $\mathcal{P}$ of coefficients of the characteristic polynomial, and how pole placement defines an affine mapping from the $\mathscr{P}$ space to the parameter space $\mathcal{K}$ of state feedback gains. The possibilities of the $\mathcal{K}$ space design are illustrated, in particular, actuator constraints and robustness with respect to measurement noise, quantization effects in the controller, sensor failures, and large plant parameter variations in known directions.

In summary,

1) $\mathcal{K}$ space design in iterative steps is readily applicable to systems with output feedback. In each design step all feedback gains except two are fixed and the influence of the remaining two gains on the pole location is easily exhibited graphically. This is a practical complement to the root locus method, which shows the influence of only one gain on the eigenvalue location. Also, large plant parameter variations in several directions can easily be tackled.

2) Computer graphics will make it possible to look at the influence of three gains at a time.

3) Algebraic conditions for arbitrary system order and circular pole regions are available. This is of particular interest for digital control. However, algorithms for the design of robust control systems based on these algebraic conditions are not yet developed.

4) For the multiinput case the formalism is available. It is, however, not yet fully understood.

Further open research problems are the design for robustness with respect to actuator failures, and a combination of parameter space methods with the Popov method for absolute stability in order to achieve robustness with respect to actuator nonlinearities of a sector type [1]. 


\section{REFERENCES}

[1] D. D. Siljak, Nonlinear Systems, The Parameter Analysis and Design. New York: Wiley, 1969.

[2] A. T. Fam and J. S. Meditch, "A canonical parameter space for linear system design," IEEE Trans. Automat. Contr., vol. AC-23, pp. 454-458, June 1978.

[3] W. Franz, "Topologie," l. Allgemeine Topologie, Sammlung Göschen, Berlin, 1965.

[4] J. Rissanen, "Control system synthesis by analogue computer based on the generalized linear feedback concept," in Proc. Int. Seminar on Analog Computation Applied to the Study of Chemical Processes. Brussels, Belgium: Presses Academiques Europeennes, Nov. 1960.

[5] J. Ackermann, Abtastregelung (Sampled-Data Control). Berlin: Springer, 1972.

[6] " "Flying qualities of piloted airplanes," MIL-F-8785B(ASG), Aug. 7. 1969 .

[7] H. R. Sirisena and S. S. Choi, "Pole placement in prescribed regions of the complex plane using output feedback," IEEE Trans. Automat. Contr., vol. AC-20, pp. 810-812, 1975.

[8] S. N. Franklin, "Design of a robust flight control system," M. S. thesis, Univ. Illinois, Urbana, 1979.

[9] J. Ackermann, "A robust control system design," in Proc. Joint Automat. Contr. Conf., Denver, CO, June 1979, pp. 877-883.
[10] "On the synthesis of linear control systems with specified characteristics," Automatica, vol. 13, pp. 89-94, 1977.

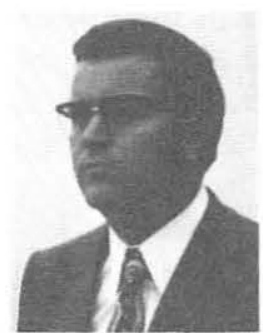

Juergen Ackermann was born in Bochum, Germany, on October 5, 1936. He received the M.S. degree in electrical engineering from the University of California, Berkeley, in 1964, the Dr.-Ing. degree from the Technische Hochschule Darmstadt in 1967, and the "Habilitation" from Technische Universitaet Muenchen in 1974.

Since 1962 he has been with the Deutsche Forschungs-und Versuchsanstalt fuer Luft-und Raumfahrt, and since 1974 he has been Director of the Institut fuer Dynamik der Flugsysteme. In 1978-1979 he was Visiting Professor at the University of Illinois. He is author of the book Abtastregelung (Berlin: Springer), Associate Editor of Automatica, and recipient of the VDE award for contributions in control engineering. His current research interests are in design of robust control systems. 